\title{
Harmine stimulates proliferation of human neural progenitors
}

\author{
Vanja Dakic ${ }^{1,2}$, Renata de Moraes Maciel ${ }^{1}$, Hannah Drummond ${ }^{1,2}$, Juliana M Nascimento ${ }^{1,3}$, Pablo Trindade \\ 1, Stevens K Rehen ${ }^{\text {Corresp. } 1,2}$ \\ 1 IDOR, D'Or Institute for Research and Education, Rio de Janeiro, RJ, Brazil \\ 2 Institute of Biomedical Sciences, Federal University of Rio de Janeiro, Rio de Janeiro, RJ, Brazil \\ 3 Department of Biochemistry and Tissue Biology / Institute of Biology, State University of Campinas (UNICAMP), Campinas, SP, Brazil \\ Corresponding Author: Stevens K Rehen \\ Email address: srehen@lance-ufrj.org
}

Harmine is the $\beta$-carboline alkaloid with the highest concentration in the psychotropic plant decoction Ayahuasca. In rodents, classical antidepressants reverse the symptoms of depression by stimulating neuronal proliferation. It has been shown that Ayahuasca presents antidepressant effects in patients with depressive disorder. In the present study, we investigated the effects of harmine in cell cultures containing human neural progenitor cells (hNPCs, 97\% nestin-positive) derived from pluripotent stem cells. After 4 days of treatment, the pool of proliferating hNPCs increased by $71.5 \%$. Harmine has been reported as a potent inhibitor of the dual specificity tyrosine-phosphorylation-regulated kinase (DYRK1A), which regulates cell proliferation and brain development. We tested the effect of analogs of harmine, an inhibitor of DYRK1A (INDY), and an irreversible selective inhibitor of monoamine oxidase (MAO) but not DYRK1A (pargyline). INDY but not pargyline induced proliferation of hNPCs similarly to harmine, suggesting that inhibition of DYRK1A is a possible mechanism to explain harmine effects upon the proliferation of hNPCs. Our findings show that harmine enhances proliferation of hNPCs and suggest that inhibition of DYRK1A may explain its effects upon proliferation in vitro and antidepressant effects in vivo. 
1 Title: Harmine stimulates proliferation of human neural progenitors

2 Authors: Vanja Dakic ${ }^{1,2}$, Renata de Moraes Maciel ${ }^{1}$, Hannah Drummond ${ }^{1,2}$,

3 Juliana M. Nascimento ${ }^{1,3}$, Pablo Trindade ${ }^{1}$, Stevens K. Rehen ${ }^{1,2, *}$

4 'D'Or Institute for Research and Education (IDOR), Rio de Janeiro, RJ, Brazil

5 2Institute of Biomedical Sciences, Federal University of Rio de Janeiro, Rio de

6 Janeiro, RJ, Brazil

7 '3epartment of Biochemistry and Tissue Biology, Institute of Biology, State

8 University of Campinas, Campinas, SP, Brazil

$9 \quad{ }^{*}$ Corresponding author: S.K.R. (srehen@lance-ufrj.org)

\section{Abstract}

12 Harmine is the $\beta$-carboline alkaloid with the highest concentration in the psychotropic plant decoction Ayahuasca. In rodents, classical antidepressants reverse the symptoms of depression by stimulating neuronal proliferation. It has been shown that Ayahuasca presents antidepressant effects in patients with depressive disorder. In the present study, we investigated the effects of harmine in cell cultures containing human neural progenitor cells (hNPCs, 97\% nestinpositive) derived from pluripotent stem cells. After 4 days of treatment, the pool of proliferating hNPCs increased by $71.5 \%$. Harmine has been reported as a potent inhibitor of the dual specificity tyrosine-phosphorylation-regulated kinase (DYRK1A), which regulates cell proliferation and brain development. We tested the effect of analogs of harmine, an inhibitor of DYRK1A (INDY), and an irreversible selective inhibitor of monoamine oxidase (MAO) but not DYRK1A 24 (pargyline). INDY but not pargyline induced proliferation of hNPCs similarly to harmine, suggesting that inhibition of DYRK1A is a possible mechanism to explain harmine effects upon the proliferation of hNPCs. Our findings show that harmine enhances proliferation of hNPCs and suggest that inhibition of DYRK1A may explain its effects upon proliferation in vitro and antidepressant effects in 29 vivo.

Introduction 
32 Throughout life, specific regions in the human adult brain continuously generate 33 neural cells from a pool of neural progenitor cells (hNPCs). Many physiological 34 and pathological events are able to control neurogenesis by modulating 35 proliferation, differentiation, maturation and integration of new-born neurons into 36 the existing circuitry (Zhao et al. 2008). This balance can be disrupted by chronic 37 stress (Egeland et al. 2015), depression (Mahar et al. 2014), aging (DeCarolis et 38 al. 2015), and neurodegenerative diseases (Winner \& Winkler 2015).

39 Classical antidepressants can reverse or block stress-induced hippocampal 40 atrophy in rodents, mostly by stimulating neuronal proliferation (Malberg et al. 41 2000)). Fluoxetine, one of the most used selective serotonin reuptake inhibitors, 42 induces proliferation of rat hypothalamic (Chen et al. 2007; Sachs \& Caron 2015; 43 Sousa-Ferreira et al. 2014)) and hippocampal neural progenitors in vitro and in 44 vivo (Chen et al. 2007; Sachs \& Caron 2015). Unfortunately, treatment with 45 classic antidepressants leads to full remission in only $50 \%$ of patients (Nestler et 46 al. 2002), causes side effects and the time required for achieving therapeutic response is usually measured in weeks. Thus, the demand for novel psychopharmacological agents able to revert depression remains significant. Beta-carbolines, a large group of indole alkaloids are widely distributed in plants. Two members of this group, harmine and harmaline, have been found in human 51 plasma after ingestion of Ayahuasca (Callaway et al. 1996), a psychotropic 52 beverage traditionally used in the Amazonian region of South America as part of 53 local religious ceremonies (Labate \& Feeney 2012).

54 Evaluation of the effects of a single dose of Ayahuasca in six volunteers with a 55 current depressive episode suggested that this plant decoction has fast-acting 56 anxiolytic and antidepressant effects (Osorio Fde et al. 2015). Moreover, the use

57 of harmine in rodents leads to the reduction of symptoms associated with 58 depression (Farzin \& Mansouri 2006) and re-establishment of normal levels of 59 hippocampal brain-derived neurotrophic factor (BDNF) (Fortunato et al. 2009).

60 Apart of these initial studies, there are no data available regarding the 61 neurogenic effects of harmine in humans. Here we examine the effects of 62 harmine on the proliferation of human neural progenitor cells derived from 
63 pluripotent stem cells. We show that harmine increased the pool of neural 64 progenitor cells and that inhibition of DYRK1 is the possible mechanism involved 65 in those proliferative effects.

66

\section{Material and methods}

68 Chemicals

69 Harmine (286044), INDY (SML1011), and pargyline hydrochloride (P8013) were 70 purchased from Sigma-Aldrich and diluted in DMSO. Subsequent dilutions were 71 made in aqueous solution. Click-it EdU kit and $\mathrm{BOBO}^{\mathrm{TM}}-3$ were purchased from 72 Thermo Fisher Scientific. All controls received an amount of vehicle equivalent to 73 drug treatment conditions and no significant difference was observed between 74 controls with (DMSO) or without vehicle.

75

76

77

\section{Human pluripotent stem cells}

Human embryonic stem cells (Fraga et al. 2011) were cultured under feeder-free culture conditions on Matrigel (BD Biosciences)-coated dishes (Corning) in Essential $8^{\mathrm{TM}}$ Medium (Thermo Fisher Scientific). Passaging was performed enzymatically using Accutase (Millipore) by splitting colonies in clumps every 4-5 days and re-plating on Matrigel-coated dishes, having their medium changed every day. All cells were maintained at $37^{\circ} \mathrm{C}$ in humidified air with $5 \% \mathrm{CO}_{2}$.

\section{Human neural progenitor cells}

To induce embryonic stem cells to direct neural differentiation, we performed an adaptation of Baharvand and co-workers protocol (Baharvand et al. 2007; Paulsen Bda et al. 2012). Briefly, 70\% confluent BR1 culture was differentiated to the neural lineage in defined adherent culture by retinoic acid and basic fibroblast growth factor (bFGF) within 18 days of culture. On the 18th day neural tube-like structures were collected and replated on dishes coated with $10 \mu \mathrm{g} / \mathrm{mL}$ of Poly-Lornithine and $2.5 \mu \mathrm{g} / \mathrm{mL}$ of laminin (Thermo Fisher Scientific). A population of hNPCs that migrated from neural tube-like structures was tested for expression 
94 supplemented with $25 \mathrm{ng} / \mathrm{mL}$ bFGF and $20 \mathrm{ng} / \mathrm{mL}$ EGF (Thermo Fisher 95 Scientific). N2B27 medium consisted of DMEM/F-12 supplemented with 1X N2, 96 1X B-27, 1\% penicillin/streptomycin (Thermo Fisher Scientific). Cells were 97 incubated at $37^{\circ} \mathrm{C}$ and $5 \% \mathrm{CO}_{2}$. Medium was replaced every other day. hNPCs 98 were expanded for no more than 5 passages.

\section{High Content Screening}

101 Cell proliferation, cell death and DNA damage experiments were performed in a 102 High Content Screening (HCS) format. hNPCs (1,500 cells/per well) were plated 103 on a multiwell $384 \mu$ Clear plate (Greiner Bio-One) coated with $100 \mu \mathrm{g} / \mathrm{mL}$ Poly-L104 ornithine and $10 \mu \mathrm{g} / \mathrm{mL}$ laminin (Thermo Fisher Scientific). After $24 \mathrm{~h}$, cells were 105 treated for 4 days in quintuplicate (five wells per condition) with harmine, INDY 106 and pargyline in N2B27 medium supplemented with bFGF and EGF. On day 4 107 cells were labelled with $10 \mu \mathrm{M}$ EdU for $2 \mathrm{~h}$ (cell proliferation) or BOBO ${ }^{\mathrm{TM}}-3$ (cell 108 death) for 30 min prior to fixation or image acquisition, respectively.

109

\section{High Content Analysis}

111 All images were acquired on Operetta high-content imaging system (Perkin 112 Elmer). For proliferation, incorporated EdU was detected with Alexa Fluor 488 113 using Click-iT EdU kit following manufacturer's instruction. Immunocytochemistry 114 for Ki-67 was performed after EdU AF488 labelling. Total number of cells was 115 calculated by nuclei stained with DAPI. S phase was determined by percentage 116 of total cells labelled with EdU. Whereas dividing cells in all phases of cell cycle, 117 exempting G0, were measured by Ki-67 positive cells as percentage of total 118 cells. Images were acquired with a 10x objective with high numerical aperture 119 (NA).

120 For cell death analysis, cells were labelled with a fluorophore dye cocktail, 121 containing the cell-permeant nuclear dye Hoechst and the cell-impermeant 122 nuclear dye BOBO ${ }^{\mathrm{TM}}-3$ in fresh N2B27 medium for 30 min at $37^{\circ} \mathrm{C}$ and $5 \% \mathrm{CO}_{2}$. 123 After incubation, the dye cocktail was replaced for new medium and live cell 124 imaging was performed using temperature and $\mathrm{CO}_{2}$ control option (TCO) of 
125 Operetta, set to $37^{\circ} \mathrm{C}$ and $5 \% \mathrm{CO}_{2}$ at $10 \mathrm{x}$ magnification. For DNA damage 126 analysis, immunocytochemistry was performed on fixed cells after 4 days of 127 treatment using $\mathrm{H} 2 \mathrm{AX}$ antibody, and images were acquired at 10x magnification. 128 All quantification analysis were normalised to the number of cells in the well 129 segmented by nucleus dyes. $\mathrm{H}_{2} \mathrm{O}_{2}$ was used as positive control for both cell 130 death and DNA damage.

131 All analyses sequences were designated by combining segmentation steps with 132 morphological and fluorescence based object characterizations using the image 133 analysis software Harmony 3.5.1 (Perkin Elmer).

134

\section{Immunocytochemistry}

$136 \mathrm{hNPCs}$ were fixed in formaldehyde $3.7 \%$ for $15 \mathrm{~min}$ at RT and permeabilized in $1370.2 \%$ Triton X-100 for 15 min Primary antibodies were incubated overnight in $2 \%$ $138 \mathrm{BSA}$ at $4^{\circ} \mathrm{C}$, following $40 \mathrm{~min}$ of $2 \% \mathrm{BSA}$ blockage. After washing with PBS, 139 secondary antibodies were incubated for $1 \mathrm{~h}$ at RT in the dark. Cells were washed 1403 times with PBS and nuclei were stained with DAPI. Coverslips were mounted 141 on slides using Aqua-Poly Mount (Polysciences) whereas cells on the 384 well 142 plates were covered with glycerol and sealed with AlumaSeal CS (Excel 143 Scientific) for image acquisition in confocal microscopy (Leica) and Operetta 144 (Perkin Elmer), respectively. Primary antibodies used: mouse anti-MAP2 (Sigma145 Aldrich), mouse anti-Ki-67 (BD Biosciences), rabbit anti-PAX6 (Santa Cruz 146 Biotechnology), rabbit anti-GFAP (Dako), rabbit anti-y-H2AX (Cell Signaling 147 Technology), rabbit anti-FOXG1 (Abcam), rabbit anti-DYRK1A (Sigma-Aldrich), 148 rabbit anti-SOX2 (Millipore), mouse anti-ß-tubulin III (Millipore), mouse anti-nestin 149 (Millipore), rabbit anti-TBR2 (Millipore). Secondary antibodies used: goat anti150 mouse Alexa Fluor 594 and goat anti-rabbit Alexa Fluor 488 (Thermo Fisher 151 Scientific). Data are expressed as relative protein expression in comparison with 152 basal protein expression in control with vehicle (DMSO).

153

\section{Statistical analysis}


155 All data are expressed as mean \pm sem. Results were accepted as statistically 156 significant at $p<0.05$, as determined using one-way ANOVA with Tukey's 157 multiple comparison test. A minimum of 10,000 hNPCs was counted per 158 condition/per experiment.

159

160 Results

161 We generated hNPCs from human embryonic stem cells using a protocol that 162 recapitulates the early steps of nervous system development (Baharvand et al. 163 2007). Characterization of specific cell markers was done on day 0 164 (Supplementary Fig. 1). More than 90\% of the hNPCs express characteristic 165 markers of neural progenitors, such as SOX2 (sex determining region Y-box 2), 166 nestin (intermediate filament protein; neuroectodermal stem cell marker), PAX6 167 (paired box 6), FOXG1 (forkhead box G1; transcriptional repressor important for 168 development of the brain and telencephalon), TBR2 (transcription factor Eomes; 169 key regulator of neurogenesis in the subventricular zone) (Fig. 1a, b, d, e, g). 170 Additionally, differentiated cells expressing $\beta$-Tubulin III (a class III member of the

$171 \beta$ tubulin protein family primarily expressed in neurons), GFAP (glial fibrillary 172 acidic protein, marker of astrocytes), or MAP2 (marker of neuronal soma and 173 dendrites) are also observed (Fig. 1b, c, g). Ninety percent of cells express the 174 dual specificity tyrosine-phosphorylation-regulated kinase 1A (DYRK1A) (Fig. 1f, $175 \mathrm{~g})$.

176 

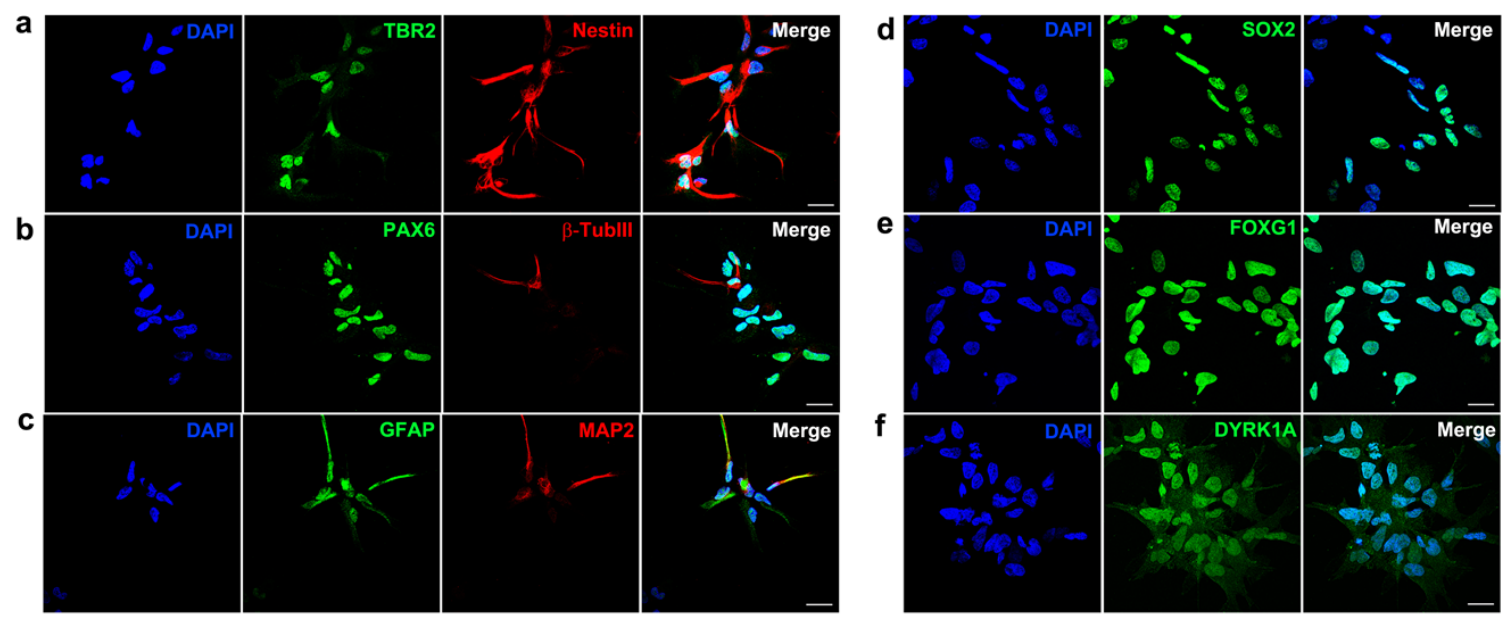

\begin{tabular}{lccccccccc}
\hline Marker & Nestin & TBR2 & PAX6 & $\beta$-Tublll & SOX2 & FOXG1 & GFAP & MAP2 & DYRK1A \\
\hline \% of positive cells & 97.08 & 91.25 & 97.25 & 0.71 & 90.97 & 98.28 & 38.99 & 5.71 & 90 \\
\hline
\end{tabular}

h
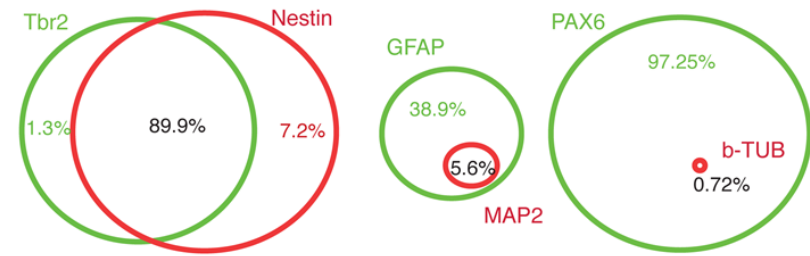

177

178

179

180

181

182

183

184

185

186

187

188

189

190

191

192

193

194

195

196

Figure 1: Characterization of neural progenitor cells derived from human embryonic stem cells. Representative images showing hNPCs stained for (a) TBR2 and Nestin, (b) PAX6 and $\beta$-Tubulin III, (c) GFAP and MAP2, (d) SOX2, (e) FOXG1, (f) DYRK1A. (g) Quantification of cell markers. (h) Venn diagram showing percentage of double stained cells. A minimum of 10,000 hNPCs was counted per marker. Scale bar: $25 \mu \mathrm{m}$.

Harmine increases proliferation of human neural progenitors: a possible role of DYRK1

Pharmacokinetic studies have shown a high variability in the bioavailability of ayahuasca alkaloids in the human plasma: 36.4-222.3 ng/mL (Callaway et al. 1996), 0.5-5 ng/mL (Yritia et al. 2002). On the other hand, in vitro studies have been using a wide spectrum of harmine concentrations, ranging from $333 \mathrm{nM}$ to 22.5 MM (Gockler et al. 2009; Hammerle et al. 2011; Martinez de Lagran et al. 2012; Mazur-Kolecka et al. 2012; Wang et al. 2015). In order to examine its effects upon proliferation, we incubated human neural cells with harmine ranging from 0.1 to $22.5 \mu \mathrm{M}$ for $96 \mathrm{~h}$ (Supplementary Figs. 1 and 2). The $7.5 \mu \mathrm{M}$ concentration of harmine was the most effective on proliferation of hNPCs (Supplementary Fig. 2), increasing the pool of proliferating cells by $71.5 \%$ (Fig. 
197 2a, b). No evidence of cell death or DNA damage in response to harmine as 198 measured by BOBO-3 (Fig. 3a, b) and H2AX labelling (Fig. 3c, d) was observed.

199 Treatment with harmine increased by $64.4 \%$ the specific pool of neural 200 progenitors, which actively participates in adult neurogenesis (Nestin and GFAP 201 labelled, Fig 2c, d) (Fukuda et al. 2003). The number of cells positive for SOX2, 202 MAP2 and FOXG1 was not altered (Supplementary Fig. 3).

a

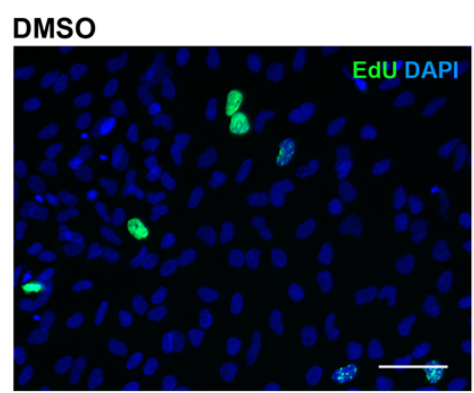

C

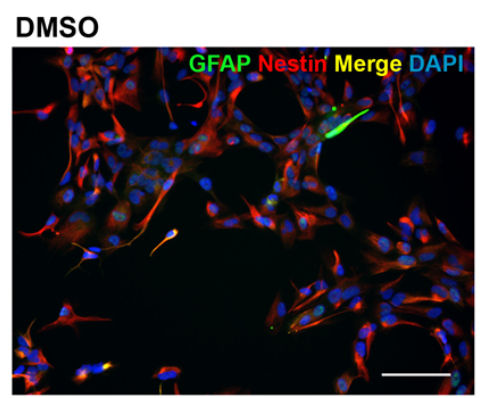

Harmine

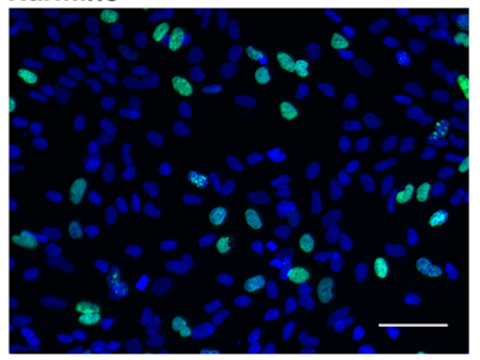

Harmine

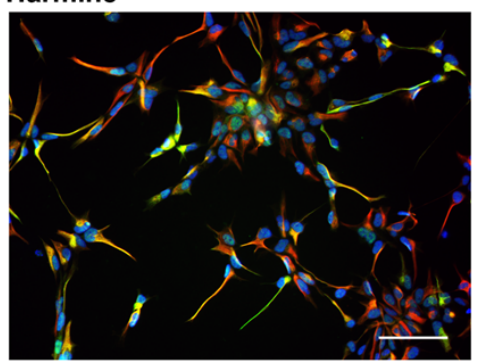

b

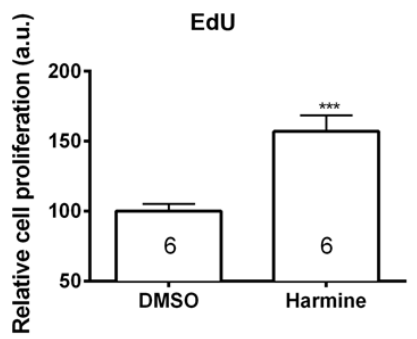

d

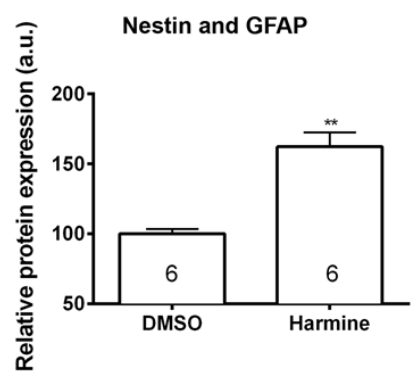

Figure 2: Quantification of the proliferation and differentiation of hNPCs after treatment with harmine. (a) Representative images of EdU staining. (b) Cell proliferation (S phase) relative to DMSO control, measured by EdU incorporation. (c) Representative images of GFAP and Nestin staining. (d) Expression of Nestin and GFAP proteins relative to DMSO control. A minimum of 10,000 hNPCs was counted per condition/per experiment. Data were analysed

210 by one-way ANOVA with Tukey's multiple comparison test, ${ }^{* *} p<0.001$, ${ }^{* * *}<0.0001$. Values represent mean \pm sem. The number inside the bar represents the number of experiments in each group. Scale bar: $100 \mu \mathrm{m}$. 
a

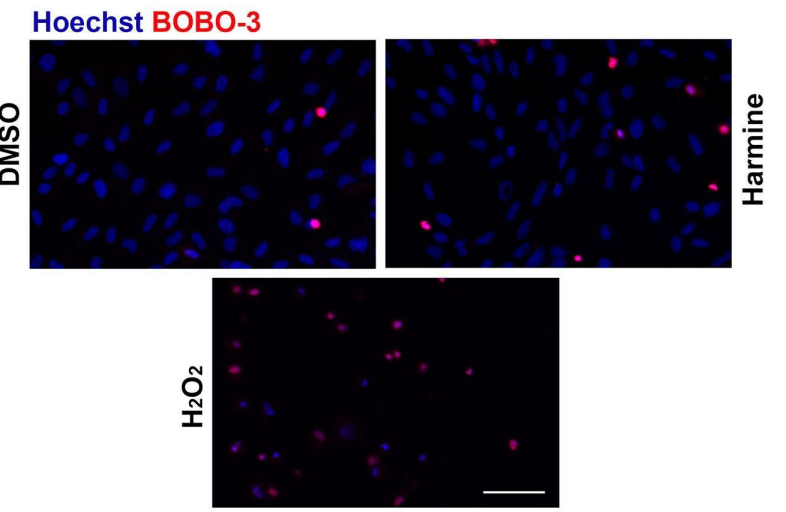

C

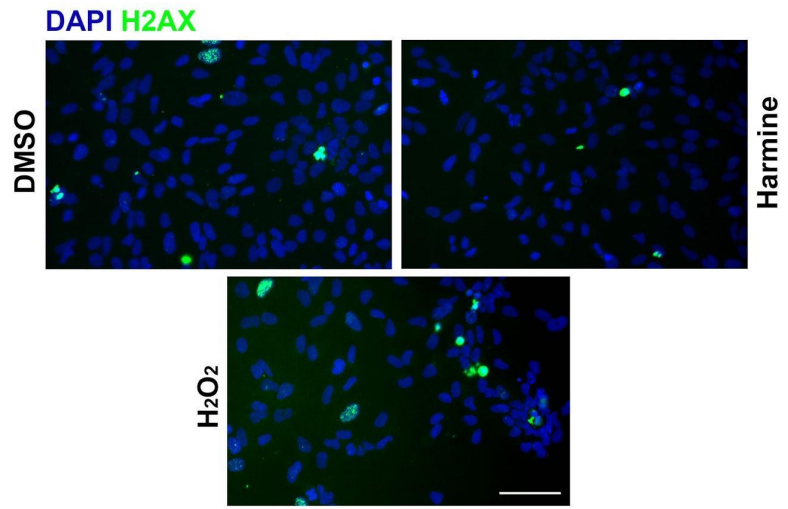

b

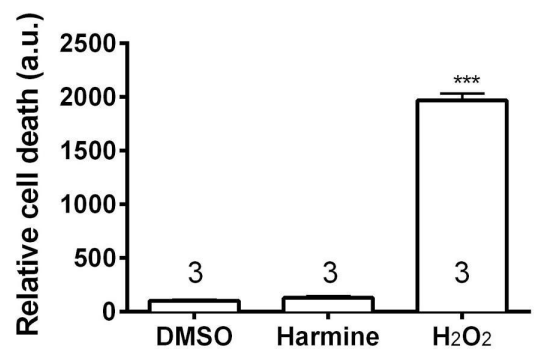

d

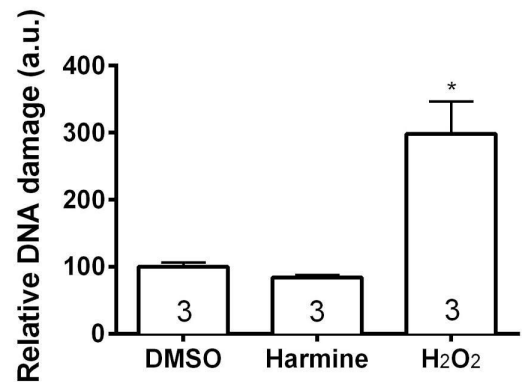

215 Figure 3: Percentage of cell death and DNA damage in neural cells treated

216 with harmine. (a) Representative immunostaining images of BOBO-3 (red)

217 positive cells. Nuclei are stained with Hoechst (blue). (b) Cell death relative to

218 DMSO control, measured as the percentage of cells stained with BOBO-3. (c)

219 Representative images of cells positive for H2AX (green). Nuclei are stained with

220 DAPI (blue). (d) Quantification of H2AX positive cells relative to DMSO control,

221 as percentage of cells stained with $\mathrm{H} 2 \mathrm{AX} . \mathrm{H}_{2} \mathrm{O}_{2}$ was used as positive control for

222 both cell death and DNA damage. A minimum of 10,000 hNPCs was counted per

223 condition/per experiment. Data were analysed by one-way ANOVA with Tukey's

224 multiple comparison test, ${ }^{*} p<0.05,{ }^{* * *}<0.0001$. Values represent mean \pm sem.

225 The number inside the bar represents the number of experiments in each group.

226 Scale bar: $100 \mu \mathrm{m}$.

228 Harmine has been shown to be an inhibitor of DYRK1A (Becker \& Sippl 2011;

229 Gockler et al. 2009), other DYRK family members (Gockler et al. 2009) and

230 monoamine oxidase (MAO) (Santillo et al. 2014). To verify whether these two

231 targets are involved in the harmine-dependent increase in neural proliferation, we

232 examined the effect of two functional analogs of harmine in human neural cells:

233 INDY $(15 \mu \mathrm{M})$, an inhibitor of DYRK1A; and pargyline (10 $\mu \mathrm{M})$, an irreversible 
234 selective inhibitor of MAO but not DYRK1A.

235 Harmine and INDY increased proliferation of hNPCs in comparison with control,

236 analysed by two different methods, while pargyline did not induce the same

237 effect. Concomitant inhibition of both DYRK1A and MAOs produced similar effect

238 as harmine or INDY alone (Fig. 4).

a

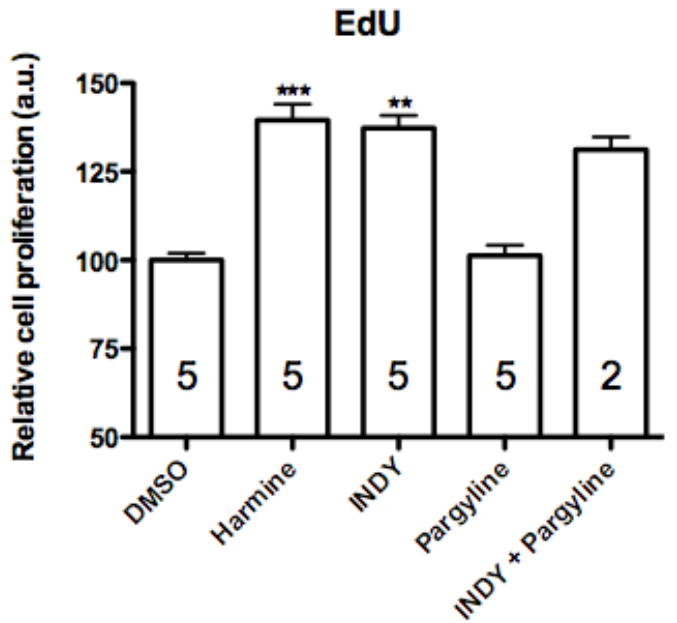

b

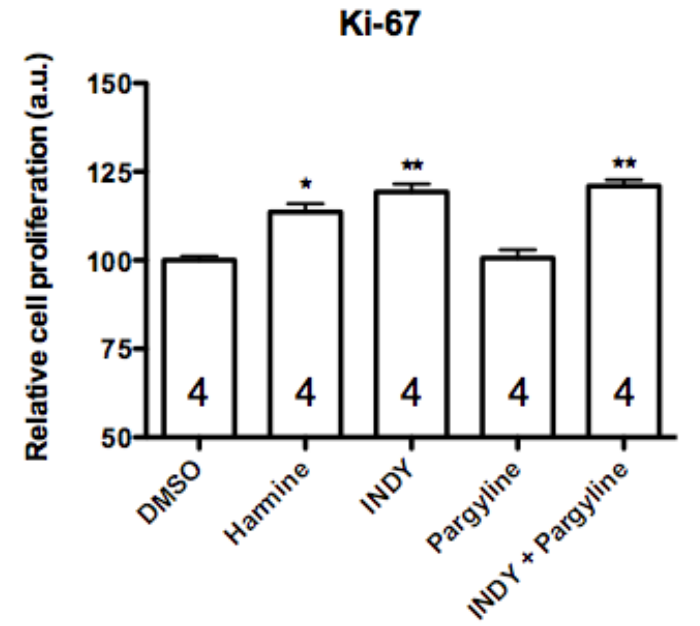

239

240

241

242

243

244

245

246

247

248

249

250

251

252

253

254

255

256

257

Figure 4: Quantification of EdU and Ki-67 labelling in human neural progenitor cells in response to harmine and its functional analogs. (a) Cell proliferation relative to DMSO control, measured by EdU incorporation. (b) Cell proliferation relative to DMSO control, measured by Ki-67. Values represent mean \pm sem. A minimum of 10,000 hNPCs was counted per condition/per experiment. Data were analysed by one-way ANOVA with Tukey's multiple comparison test, ${ }^{*} p<0.05,{ }^{* *} p<0.001,{ }^{* * *}<0.0001$. Values represent mean \pm sem. The number inside the bar represents the number of experiments in each group.

\section{Discussion}

The use of in vitro models can potentially clarify mechanisms related to proliferation and differentiation of new-born neurons (Cai \& Grabel 2007), which happens massively during embryogenesis but also later in specific brain regions. Numerous studies have shown a close relationship between disturbed adult neurogenesis and depression, highlighting the need for more efficacious and faster-acting treatments. Thus, the aim of this study was to investigate the effects of harmine, a compound with potential antidepressant properties, on human neural progenitor cells derived from pluripotent stem cells. Here we show that 
259 harmine increases proliferation of hNPCs positive for both nestin and GFAP in 260 vitro, having inhibition of DYRK1 as the possible mechanism.

261 We describe, in cell cultures containing more than $90 \%$ of hNPCs, that treatment 262 with harmine increased proliferation without DNA damage or cell death. These 263 results are consistent with findings published by Hämmerle and others (2011), in 264 which exposure of chick embryos to harmine resulted in a strong increase in 265 BrdU incorporation and number of mitotic cells in the spinal cord.

266 Interestingly, harmine treatment also increased the number of early progenitor 267 cells, stained with both GFAP and Nestin. At first, this result suggests that 268 harmine drives the differentiation of hNPCs into radial glial cells, which are the 269 major source of neuronal and glial progenitors in the developing brain (Gotz \& 270 Barde 2005). Similarly, in the adult hippocampus of rodents, these neural 271 precursors are responsible for late neurogenesis and gliogenesis (Zhao et al. 272 2008). In this context, levels of GFAP tend to increase on cells that shifted to a 273 glial fate. On the other hand, despite being GFAP+ cells, at this developmental 274 stage, these progenitors can also shift to a phenotype PSA-NCAM+, which can 275 give rise to neurons (Fukuda et al. 2003). In the light of this evidence, we suggest 276 that harmine could potentiate proliferation of radial glia-like cells 277 (GFAP+/Nestin+) derived from hNPCs, which are capable to generate both 278 neurons and astrocytes. Further experiments should be done in order to confirm 279 the terminal fate of harmine-treated hNPCs.

280 Harmine has been described as an inhibitor of MAO and DYRK1A (Gockler et al. 281 2009; Santillo et al. 2014). MAO inhibition increases serotonergic 282 neurotransmission in the adult brain (Finberg, 2014), which is a key component 283 of the classical antidepressant action. The main outcome of treatment with MAO 284 inhibitors is upregulation of cell proliferation and neurogenesis in the 285 hippocampus (Manev et al. 2001). On the other hand, it has been reported that 286 mice lacking MAO presented reduced proliferation of neural progenitor cells 287 (Cheng et al. 2010). In order to verify if MAO inhibition plays a role in harmine288 induced proliferation, we used the specific MAO inhibitor, pargyline. Pargyline did 289 not alter the levels of harmine-induced proliferation. While these results indicate 
290 that MAO is not involved in the proliferative effects of harmine, we can not rule

291 out that the lack of effect of pargyline is due to the absence of MAO in neural 292 progenitor cells. In fact, MAO-dependent changes in NPCs proliferation were 293 described late in embryonic development, mediated by serotonin (Cheng et al. 294 2010).

295 On the other hand, treatment with INDY, an inhibitor of DYRK1, induced 296 proliferation similarly to that observed with harmine. These results are consistent 297 with the findings of Wang and collaborators, where harmine-dependent DYRK1A 298 inhibition increases the proliferation of pancreatic beta cells (Zhou et al. 2015). 299 Since both harmine and INDY have multiple targets on DYRK family, including 300 regulators of cell cycle (Becker \& Sippl 2011), we should consider other potential 301 players of INDY- and harmine-mediated increase in proliferation.

302 INDY can also inhibit DYRK1A closely related kinases, DYRK1B and DYRK2. In 303 cancer studies DYRK1B and DYRK2 have been described as modulators of 304 proliferation (Adayev et al. 2011; Zhou et al. 2015). In fact, harmine also inhibits 305 DYRK1B and DYRK2, but the efficiency of this inhibition is, respectively, 5- and 306 50-fold lower in comparison to DYRK1A (Adayev et al. 2011; Bain et al. 2007; 307 Gockler et al. 2009). Thus, DYRK1A emerges as the major candidate for 308 mediating the increase in proliferation seen in this study. Also, it was shown that 309 DYRK1A directly phosphorylates p53 and leads to the induction of p53 target 310 genes, attenuating proliferation of rat and human neural progenitor cells (Park et 311 al. 2010). Further studies are needed to reveal other aspects of human cell 312 proliferation stimulated by harmine.

313 It is worth to mention that DYRK1A can also modulate migration (Pons-Espinal et 314 al. 2013) and differentiation (Kurabayashi \& Sanada 2013; Yabut et al. 2010) of 315 neural progenitors. Neurogenesis contemplates the interplay of proliferation, 316 migration and differentiation. While our data show that harmine stimulates 317 proliferation of hNPCs, further studies upon migration and differentiation may 318 clarify whether neurogenesis (or gliogenesis) are indeed increased by harmine in 319 hNPCs.

320 Taken together our results suggest that harmine exert proliferative effects in 
321 human neural progenitors, particularly in radial glia-like cells (GFAP+/Nestin+),

322 by inhibiting DYRK1A. These findings shed light on the possible mechanisms

323 behind the antidepressant effects of Ayahuasca described in patients.

324

325

326

327

328

329

330

331

332

333

334

335

336

337

338

339

340

341

342

343

344

345

346

347

348

349

350

351

352

353

354

355

356

357

358

359

360

361

362

363

\section{Abbreviations}

hNPCs - human neural progenitors; DYRK - dual-specificity tyrosine phosphorylation-regulated kinase; MAO - monoamine oxidase; GFAP - Glial fibrillary acidic protein; MAP2 - Microtubule-Associated Protein 2;

\section{Acknowledgements}

This work is part of the PhD thesis of VD. We thank Ismael Gomes, Marcelo Costa and Igor Lima da Silva for technical assistance and Dr. Mauro de Freitas Rebelo for assistance with statistical analysis.

\section{References}

Adayev T, Wegiel J, and Hwang YW. 2011. Harmine is an ATP-competitive inhibitor for dual-specificity tyrosine phosphorylation-regulated kinase $1 \mathrm{~A}$ (Dyrk1A). Arch Biochem Biophys 507:212-218. 10.1016/j.abb.2010.12.024

Baharvand H, Mehrjardi NZ, Hatami M, Kiani S, Rao M, and Haghighi MM. 2007. Neural differentiation from human embryonic stem cells in a defined adherent culture condition. Int $J$ Dev Biol 51:371-378. 10.1387/ijdb.72280hb

Bain J, Plater L, Elliott M, Shpiro N, Hastie CJ, McLauchlan H, Klevernic I, Arthur JS, Alessi DR, and Cohen P. 2007. The selectivity of protein kinase inhibitors: a further update. Biochem J 408:297-315. 10.1042/BJ20070797

Becker W, and Sippl W. 2011. Activation, regulation, and inhibition of DYRK1A. FEBS J 278:246-256. 10.1111/j.1742-4658.2010.07956.x

Cai C, and Grabel L. 2007. Directing the differentiation of embryonic stem cells to neural stem cells. Dev Dyn 236:3255-3266. 10.1002/dvdy.21306

Callaway JC, Raymon LP, Hearn WL, McKenna DJ, Grob CS, Brito GS, and Mash DC. 1996. Quantitation of N,N-dimethyltryptamine and harmala alkaloids in human plasma after oral dosing with ayahuasca. J Anal Toxicol 20:492-497.

Chen SJ, Kao CL, Chang YL, Yen CJ, Shui JW, Chien CS, Chen IL, Tsai TH, Ku $\mathrm{HH}$, and Chiou SH. 2007. Antidepressant administration modulates neural stem cell survival and serotoninergic differentiation through bcl-2. Curr Neurovasc Res 4:19-29.

Cheng A, Scott AL, Ladenheim B, Chen K, Ouyang X, Lathia JD, Mughal M, Cadet JL, Mattson MP, and Shih JC. 2010. Monoamine oxidases regulate telencephalic neural progenitors in late embryonic and early postnatal 
development. J Neurosci 30:10752-10762. 10.1523/JNEUROSCI.203710.2010

DeCarolis NA, Kirby ED, Wyss-Coray T, and Palmer TD. 2015. The Role of the Microenvironmental Niche in Declining Stem-Cell Functions Associated with Biological Aging. Cold Spring Harb Perspect Med 5. 10.1101/cshperspect.a025874

Egeland M, Zunszain PA, and Pariante CM. 2015. Molecular mechanisms in the regulation of adult neurogenesis during stress. Nat Rev Neurosci 16:189200. $10.1038 / \mathrm{nrn} 3855$

Farzin D, and Mansouri N. 2006. Antidepressant-like effect of harmane and other beta-carbolines in the mouse forced swim test. Eur Neuropsychopharmacol 16:324-328. 10.1016/j.euroneuro.2005.08.005

Finberg JP. 2014. Update on the pharmacology of selective inhibitors of MAO-A and MAO-B: Focus on modulation of CNS monoamine neurotransmitter release. Pharmacol Ther. 143(2):133-52. 10.1016/j.pharmthera.2014.02.010.

Fortunato JJ, Reus GZ, Kirsch TR, Stringari RB, Stertz L, Kapczinski F, Pinto JP, Hallak JE, Zuardi AW, Crippa JA, and Quevedo J. 2009. Acute harmine administration induces antidepressive-like effects and increases BDNF levels in the rat hippocampus. Prog Neuropsychopharmacol Biol Psychiatry 33:1425-1430. 10.1016/j.pnpbp.2009.07.021

Fraga AM, Sukoyan M, Rajan P, Braga DP, laconelli A, Jr., Franco JG, Jr., Borges E, Jr., and Pereira LV. 2011. Establishment of a Brazilian line of human embryonic stem cells in defined medium: implications for cell therapy in an ethnically diverse population. Cell Transplant 20:431-440. $10.3727 / 096368910 \times 522261$

Fukuda S, Kato F, Tozuka Y, Yamaguchi M, Miyamoto Y, and Hisatsune T. 2003. Two distinct subpopulations of nestin-positive cells in adult mouse dentate gyrus. J Neurosci 23:9357-9366.

Gockler N, Jofre G, Papadopoulos C, Soppa U, Tejedor FJ, and Becker W. 2009. Harmine specifically inhibits protein kinase DYRK1A and interferes with neurite formation. FEBS $J$ 276:6324-6337. 10.1111/j.17424658.2009.07346.x

Gotz M, and Barde YA. 2005. Radial glial cells defined and major intermediates between embryonic stem cells and CNS neurons. Neuron 46:369-372. 10.1016/j.neuron.2005.04.012

Hammerle B, Ulin E, Guimera J, Becker W, Guillemot F, and Tejedor FJ. 2011. Transient expression of Mnb/Dyrk1a couples cell cycle exit and differentiation of neuronal precursors by inducing p27KIP1 expression and suppressing NOTCH signaling. Development 138:2543-2554. 10.1242/dev.066167

Kurabayashi N, and Sanada K. 2013. Increased dosage of DYRK1A and DSCR1 delays neuronal differentiation in neocortical progenitor cells. Genes Dev 27:2708-2721. 10.1101/gad.226381.113 
408 Labate BC, and Feeney K. 2012. Ayahuasca and the process of regulation in

409

410

411

412

413

414

415

416

417

418

419

420

421

422

423

424

425

426

427

428

429

430

431

432

433

434

435

436

437

438

439

440

441

442

443

444

445

446

447

448

449

450

451

452

453

Brazil and internationally: implications and challenges. Int J Drug Policy 23:154-161. 10.1016/j.drugpo.2011.06.006

Mahar I, Bambico FR, Mechawar N, and Nobrega JN. 2014. Stress, serotonin, and hippocampal neurogenesis in relation to depression and antidepressant effects. Neurosci Biobehav Rev 38:173-192. 10.1016/j.neubiorev.2013.11.009

Malberg JE, Eisch AJ, Nestler EJ, and Duman RS. 2000. Chronic antidepressant treatment increases neurogenesis in adult rat hippocampus. J Neurosci 20:9104-9110.

Manev H, Uz T, Smalheiser NR, and Manev R. 2001. Antidepressants alter cell proliferation in the adult brain in vivo and in neural cultures in vitro. Eur $J$ Pharmacol 411:67-70.

Martinez de Lagran M, Benavides-Piccione R, Ballesteros-Yanez I, Calvo M, Morales M, Fillat C, Defelipe J, Ramakers GJ, and Dierssen M. 2012. Dyrk1A influences neuronal morphogenesis through regulation of cytoskeletal dynamics in mammalian cortical neurons. Cereb Cortex 22:2867-2877. 10.1093/cercor/bhr362

Mazur-Kolecka B, Golabek A, Kida E, Rabe A, Hwang YW, Adayev T, Wegiel J, Flory M, Kaczmarski W, Marchi E, and Frackowiak J. 2012. Effect of DYRK1A activity inhibition on development of neuronal progenitors isolated from Ts65Dn mice. $J$ Neurosci Res 90:999-1010. 10.1002/jnr.23007

Nestler EJ, Barrot M, DiLeone RJ, Eisch AJ, Gold SJ, and Monteggia LM. 2002. Neurobiology of depression. Neuron 34:13-25.

Osorio Fde L, Sanches RF, Macedo LR, Santos RG, Maia-de-Oliveira JP, Wichert-Ana L, Araujo DB, Riba J, Crippa JA, and Hallak JE. 2015. Antidepressant effects of a single dose of ayahuasca in patients with recurrent depression: a preliminary report. Rev Bras Psiquiatr 37:13-20. 10.1590/1516-4446-2014-1496

Park J, Oh Y, Yoo L, Jung MS, Song WJ, Lee SH, Seo H, and Chung KC. 2010. Dyrk1A phosphorylates p53 and inhibits proliferation of embryonic neuronal cells. J Biol Chem 285:31895-31906. 10.1074/jbc.M110.147520

Paulsen B da S, de Moraes Maciel R, Galina A, Souza da Silveira M, dos Santos Souza C, Drummond H, Nascimento Pozzatto E, Silva H, Jr., Chicaybam L, Massuda R, Setti-Perdigao P, Bonamino M, Belmonte-de-Abreu PS, Castro NG, Brentani H, and Rehen SK. 2012. Altered oxygen metabolism associated to neurogenesis of induced pluripotent stem cells derived from a schizophrenic patient. Cell Transplant 21:1547-1559. 10.3727/096368911X600957

Pons-Espinal M, Martinez de Lagran M, and Dierssen M. 2013. Environmental enrichment rescues DYRK1A activity and hippocampal adult neurogenesis in TgDyrk1A. Neurobiol Dis 60:18-31. 10.1016/j.nbd.2013.08.008

Sachs BD, and Caron MG. 2015. Chronic fluoxetine increases extra-hippocampal neurogenesis in adult mice. Int $J$ Neuropsychopharmacol 18. 10.1093/ijnp/pyu029 
454 Santillo MF, Liu Y, Ferguson M, Vohra SN, and Wiesenfeld PL. 2014. Inhibition

455

456

457

458

459

460

461

462

463

464

465

466

467

468

469

470

471

472

473

474

475

476

477

478

479

480

481

482

483

484

485 of monoamine oxidase (MAO) by beta-carbolines and their interactions in live neuronal (PC12) and liver (HuH-7 and $\mathrm{MH} 1 \mathrm{C} 1)$ cells. Toxicol In Vitro 28:403-410. 10.1016/j.tiv.2013.12.006

Sousa-Ferreira L, Aveleira C, Botelho M, Alvaro AR, Pereira de Almeida L, and Cavadas C. 2014. Fluoxetine induces proliferation and inhibits differentiation of hypothalamic neuroprogenitor cells in vitro. PLoS One 9:e88917. 10.1371/journal.pone.0088917

Wang P, Alvarez-Perez JC, Felsenfeld DP, Liu H, Sivendran S, Bender A, Kumar A, Sanchez R, Scott DK, Garcia-Ocana A, and Stewart AF. 2015. A highthroughput chemical screen reveals that harmine-mediated inhibition of DYRK1A increases human pancreatic beta cell replication. Nat Med 21:383-388. 10.1038/nm.3820

Winner B, and Winkler J. 2015. Adult neurogenesis in neurodegenerative diseases. Cold Spring Harb Perspect Biol 7:a021287. 10.1101/cshperspect.a021287

Yabut O, Domogauer J, and D'Arcangelo G. 2010. Dyrk1A overexpression inhibits proliferation and induces premature neuronal differentiation of neural progenitor cells. $J$ Neurosci 30:4004-4014. 10.1523/JNEUROSCI.4711-09.2010

Yritia M, Riba J, Ortuno J, Ramirez A, Castillo A, Alfaro Y, de la Torre R, and Barbanoj MJ. 2002. Determination of N,N-dimethyltryptamine and betacarboline alkaloids in human plasma following oral administration of Ayahuasca. J Chromatogr B Analyt Technol Biomed Life Sci 779:271-281.

Zhao C, Deng W, and Gage FH. 2008. Mechanisms and functional implications of adult neurogenesis. Cell 132:645-660. 10.1016/j.cell.2008.01.033

Zhou N, Yuan S, Wang R, Zhang W, and Chen JJ. 2015. Role of dual specificity tyrosine-phosphorylation-regulated kinase 1B (Dyrk1B) in S-phase entry of HPV E7 expressing cells from quiescence. Oncotarget 6:30745-30761. 10.18632/oncotarget.5222 